\title{
Evidence Based Development of Herbal Medicines
}

\author{
Bilge Sener* \\ Department of Pharmacognosy, Turkey
}

Received: 眥: November 30, 2018; Published: 制: December 14, 2018

*Corresponding author: Bilge SENER, Gazi University, Faculty of Pharmacy, Department of Pharmacognosy, Ankara, Turkey

Abbreviations: EMA: European Medicines Agency; EU: European Union; THMP: Traditional Herbal Medicinal Products

\section{Short Communication}

Herbal medicines play an important role in the primary health care of billions of people around the globe. Herbal medicines are also increasingly becoming more popular in wealthier developed nations in the recent past as people seek natural alternatives and herbal medicines become globalized. However, there are serious concerns regarding the quality, safety and claimed efficacy and consistency of most of the herbal medicines purchased around the world. There is no concerted effort visible in the research, manufacturing and regulatory arenas to ameliorate these concerns and developer herbal medicines as a real therapeutic agent in the sense of modern science for both the practitioner and the patient. Herbal medicinal products named as "Phytomedicines" exhibit a variety of biological activities on human health and used in phytotherapy. One of the major problems with the formulation of herbal medicines is the quality control of plant material to ensure its efficacy and safety.

The advantage of phytotherapy is the availability of a wide group of medicinal plants and preparations that have been used over the centuries almost exclusively on the basis of empirical evidence. The current availability of high-tech methods allows researchers to optimize the effectiveness, standardization and clinical testing of these traditional medicines to meet today's international standards. These range from the control of regulatory processes by Health Authorities is essential for human life. Therefore, herbal medicinal products are also subject to the same legislative controls as other medicines.

\section{Quality Issue}

The rise in the use of herbal medicines has led to the various forms of quality deterioration and adulteration which can often lead to fatal consequences for the end user. Therefore, it is important to standardize and ensure the quality of herbal medicines. Liquid chromatography electrospray ionization tandem mass spectrometry offers fast, sensitive and high-throughput tool for the identification and quantification of natural products in complex mixtures. Quality control of herbal medicines requires the identification and quantification of multiple chemical constituents present in the mixture. Since a single chemical marker is not sufficient to establish the quality profile, but a quantitative profile of multiple constituents needs to be established. There are specific requirements for herbal medicinal products in terms of their quality. These requirements are independent from the legal status [1]. Thus, the same quality standards equally apply to herbal products based on clinical evidence and traditional herbal medicinal products. The basic principle is that the quality of herbal medicinal products is intrinsically associated with the quality standard of the herbal substances and/or herbal preparations.

Furthermore, the herbal substance or herbal preparation in its entirety is regarded as the active substance. Consequently, the determination of the content of marker(s) or constituents with known therapeutic activity is not sufficient for the quality control of herbal medicinal products. Specific quality requirements include thorough product characterization, adherence to the Good Agricultural and Collection Practices, Good Manufacturing Practices and validated manufacturing process, e.g., raw material testing, inprocess testing, fingerprint characterization etc. Quality control of herbal medicinal products is primarily intended to define the quality of the herbal substance/preparation and herbal medicinal product rather than to establish full characterization. The European Pharmacopoeia (Ph. Eur.) provides a legal and scientific reference for the quality control of medicines [2]. The requirements for a specific herbal drug are prescribed in the corresponding individual monograph and the relevant general monographs. Criteria for pesticides and heavy metals for example are defined in the general monograph on herbal drugs. 
The Ph. Eur. also provides general methods including methods for determination of aflatoxins B1 and ochratoxin A. Screening methods for aristolochic acids are applied for herbal drugs that may be subject to adulteration or substitution with plant material containing aristolochic acids. The Ph. Eur. also collaborate in many areas with the European Medicines Agency (EMA) to ensure close collaboration as regards the respective work programmers and approach. Pharmaceutical quality of medicinal product is the basis for ensuring safe and effective medicines. The basic principles governing the assurance of the quality of medicinal products in the European Union are primarily defined in the amended Directive 2001/83/EC and Directive 2003/63/EC. Quality requirements of herbal medicinal products are also laid down in scientific guidelines. Scientific guidelines provide a basis for practical harmonization of how the competent authorities of EU Member States interpret and apply the detailed requirements for the demonstration of quality laid down in regulations and directives.

Detailed quality requirements for herbal medicinal products on the European market are contained in European Union (EU) pharmaceutical legislation. They include a system of manufacturing authorizations' which ensures that all herbal medicinal products on the European market are manufactured/imported only by authorised manufacturers, whose activities are regularly inspected by the competent authorities. Additionally, as starting materials only active substances are allowed which have been manufactured in accordance with the GMP for starting materials as adopted by the Community.

\section{Efficacy Issue}

Herbal medicinal products for which the efficacy is demonstrated by results of a set of clinical trials that are in conformity with the relevant guidelines of the therapeutic area in question. This regulatory pathway to obtain a marketing authorisation for a new medicinal product (new chemical entity) is open to herbal medicinal products, but the examples are in reality few. According to Directive 2004/24/EC which amended the basic legislation laid down in Directive 2001/83/EC a new European legislation on herbal medicinal products was developed, in order to harmonize the use of herbal medicinal products in Member States of the European Union. The objective of this legislation was to ensure the future existence of such products and to consider particular characteristics during the assessment of their quality, efficacy and safety, having defined two categories for herbal medicines:

a) Well-established use herbal medicinal products, which can be granted a marketing authorization,

b) Traditional herbal medicinal products which can be granted a registration based on their longstanding safe and efficient use.

Since 2004 the regulatory framework within the European Union has a specific assessment procedure for herbal medicinal products, with a medicinal use based on traditional practice. The main requirement concerning the traditional use is focused on the period of time for medical use: at least 30 years, including 15 years in the EU. In addition to requirements for quality and safety, an evaluation of pharmacological effects or efficacy based on long-standing use, is a main objective. "Traditional Use" however encompasses European, and non-European traditional use.

\section{Safety Evaluation}

Each application for authorisation of a medicinal product must be accompanied by the particulars and documents referred to in Directive 2001/83/EC on the Community code relating to medicinal products for human use. Details on the documentation needed for traditional herbal medicinal products (THMP) are given in article $16 \mathrm{c}$ of the above mentioned Directive. It is pointed out that a bibliographic review of safety data together with an expert report and additional data, if necessary, are required within the "Non-clinical Overview". These range from the control of regulatory processes by Health Authorities is essential for human life. Therefore, herbal medicinal products are also subject to the same legislative controls as other medicines. There is neither a globally consented terminology nor a harmonized regulatory approach. Nevertheless, it is common sense that quality, efficacy and safety should be assessed following scientific standards, addressing particulars and considering an adequate level of risk management.

A global market for traditional medicines is emerging, if not already existing. Therefore, a constructive communication about regulatory systems for herbal and traditional medicinal products should be enforced. Best practice standards might be developed according to current scientific knowledge in order to improve mutual acceptance of data, sets of monographs and assessments. Overall, a convergence of the diverse regulatory systems might save resources and lead to an adequate availability of herbal and traditional medicinal products to the patients without neglecting public health. The regulatory systems for herbal medicines should be enforced. Best practice standards might be developed according to current scientific knowledge in order to improve mutual acceptance of data, sets of monographs and assessments. The assessment of the efficacy of phytomedicines by placebo-controlled double-blind trials with bioavailability and pharmacokinetic studies along with Phyto pharmacovigilance are the main key for a rational and fully accepted phytotherapy.

Therefore, the assurance of Phyto preparation quality by standardization will be claimed. Safety is the most important and self-evident precondition for the development of phytomedicines and it must be guaranteed through toxicological investigations. The assessment of the efficacy of phytomedicines by placebo-controlled double-blind trials with bioavailability and pharmacokinetic studies along with pharmacovigilance holds the main key for a rational and fully accepted phytotherapy. All these tasks can be achieved only through interdisciplinary and international cooperation.

\section{References}

1. (2011) EMEA, Guideline on Specifications: Test Procedures and Acceptance Criteria for Herbal Drugs, Herbal Drug Preparatios and Herbal Medicinal Products/Traditional Herbal Medicinal Products. EMEA/CPMP/QWP/2820/00 and (EMEA/CVMP/815/00) Rev. 1 European Medicines Agency, London.

2. Dennis VC Awang (2000) PDR for Herbal Medicines. ( $2^{\text {nd }}$ Edn) Thomson Medical Economics Montvale, New Jersey, USA, 58: 75-76. 
ISSN: 2574-1241

DOI: 10.26717/BJSTR.2018.12.002206

Bilge SENER. Biomed J Sci \& Tech Res

(C) (i) This work is licensed under Creative

Submission Link: https://biomedres.us/submit-manuscript.php

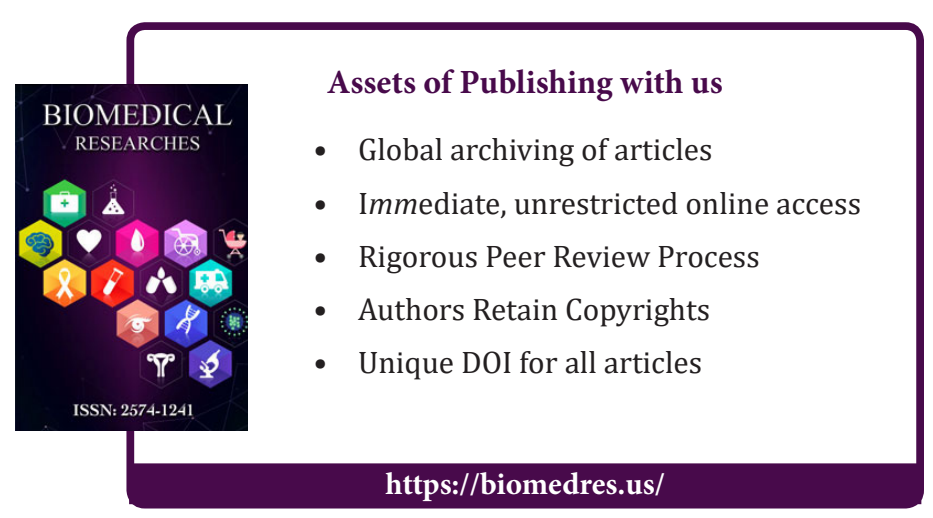

